are Doing. The project commenced in 2014 and continues to develop. There have been significant changes in the way the Unit operates alongside increased staff morale, enthusiasm, ownership and pride in the environment and maintaining and improving standards

Aims The ultimate aim of the Productive Ward foundation modules is to release time to care by ensuring efficient systems are in place in a well organised environment

Methods A project lead planned and trained three groups to lead each module. Each group nominated a group lead who reported back to the project manager quarterly. Quarterly reports were included in the annual audit plan for Dorothy House. A ward vision was produced and clearly displayed outlining out core values. A project board was introduced to demonstrate progress and maintain momentum. Before and after photographs were taken to demonstrate improvements. Once an area was completed standards were set and regular audits commenced to ensure compliance and sustainability.

Conclusions Patient Status at a Glance: An electronic whiteboard has been introduced that informs handovers and MDT meetings. This can be accessed via the desk top for all clinical teams connected with IPU. It records a variety of patient information and has the ability to aid in reporting and audit

Well Organised Ward: Each clinical area was assessed using the $5 \mathrm{~S}$ tool involving to sort, set, shine, standardise and sustain. We worked with the three second rule where any item needed could be found within three seconds of entering the room.

Knowing How we are Doing: Friends and family feedback, suggestion boxes and safety crosses were introduced. The safety crosses monitor any incidences or near misses related to pressure ulcers, falls or medication errors

\section{P-75 DEVELOPMENT OF A PALLIATIVE CARE D.R.E.A.M. AIDE MEMOIRE TO HELP IN COMPLEX DECISION-MAKING AND ASSESSMENTS IN THE PALLIATIVE CARE ARENA}

${ }^{1,2}$ Anthony Thompson. 'Willowbrook Hospice; ${ }^{2}$ St Helens and Knowsley Trust

\subsection{6/bmjspcare-2016-001245.99}

Specialist palliative care units are evolving. The ability to be more reactive in managing medical crises has enabled hospices to treat conditions such as pneumonia with intravenous antibiotics rather than transferring them to an acute trust.

With this greater ability comes greater responsibility. For these decisions to be made correctly and in conjunction with the patient and family, there is a need for highly coordinated and effective multidisciplinary team working.

With hospices becoming more involved with training of doctors, this brings huge benefits and also challenges to the units. Trainees in palliative medicine are at varying levels of training, and work closely with clinical assistants and specialty doctors.

This rich tapestry of the medical team means we need to have safe and effective handovers and medical decision making processes that are transparent and communicated properly.

Therefore, in an easy to remember format, I have devised the D.R.E.A.M. to act as a prompt for important palliative care issues that need to be considered.

It is a way of prompting both the clinician, and the multi-disciplinary team to consider the best care of the patient in a structured way.
The DREAM is completed on admission, and twice weekly within the wider MDT ward rounds.

D Discharge planning and Discussions

Careful and sensitive Discussions, Documented, Discharge planning.

$\mathrm{R}$ Resuscitation Status

Ensure this is considered on admission, unified/Hospice DNACPR documented.

E Escalation of Care Decisions

Decisions on level of treatment planned e.g. IV antibiotics, transfer out.

A Anti-coagulation/Advance Care Planning (ACP)

Prompt for medical decision making process. Evidence to support use of prophylactic heparin exists in the Palliative arena. Prompt to record and share ACP discussions.

M Mental Capacity Act (MCA)

Prompt to consider Capacity and all aspects of the Act, including DOLS, LPA and ADRTs.

\section{P-76 OFFERING AN ALCOHOL DRINK SERVICE IN A HOSPICE: ITS MEANING FOR PATIENTS}

1,2Bridget Taylor. 'Sir Michael Sobell House, Oxford, UK; ${ }^{2}$ Oxford Brookes University, Oxford, UK

10.1136/bmjspcare-2016-001245.100

Offering an alcoholic drink to patients before their lunch and evening meal is an established service in many hospice in-patient and day service units. The appropriateness of this long-established service at Sobell House has recently been a point of discussion among staff. Concerns have been expressed about the appropriateness of promoting alcohol, and its potential for harm. These discussions prompted an evaluation of this service from the patients' perspective.

A patient experience survey was conducted during January 2016, which included several questions about this alcoholic drink service. Every patient attending day service was invited to complete an anonymous questionnaire on their experience of the care received. The same questionnaire was given to patients on the ward (or their family member if they were unable to complete it), and posted to a random sample of community patients. A total of 71 completed questionnaires were returned, giving a response rate of $33 \%$.

The response to this survey has shown that the alcoholic drink service is valued by both users and non-users. 59\% of respondents explicitly endorsed the value of the service. Of the remainder, all except one were equivocal or did not comment. Respondents describe it as a valued service, which provides social benefits and a sense of normality in an institutional environment. It is also seen to convey a sense of valuing the individual as a person rather than a patient.

This survey provides evidence for the value of an alcoholic drink service for patients. As a result, the hospice is clearer about its benefit and more aware of potential concerns. An action plan has been developed to respond to patients' concerns about its cost to the charity, and to ensure that quality non-alcoholic drinks are also available. 